\title{
T-FUNCTIONS REVISITED: NEW CRITERIA FOR BIJECTIVITY/TRANSITIVITY
}

\author{
VLADIMIR ANASHIN, ANDREI KHRENNIKOV, AND EKATERINA YUROVA
}

\begin{abstract}
The paper presents new criteria for bijectivity/transitivity of Tfunctions and a fast knapsack-like algorithm of evaluation of a T-function. Our approach is based on non-Archimedean ergodic theory: Both the criteria and algorithm use van der Put series to represent 1-Lipschitz $p$-adic functions and to study measure-preservation/ergodicity of these.
\end{abstract}

\section{INTRODUCTION}

For years linear feedback shift registers (LFSRs) over a 2-element field $\mathbb{F}_{2}$ have been one of the most important building blocks in keystream generators of stream ciphers. LFSRs can easily be designed to produce binary sequences of the longest period (that is, of length $2^{k}-1$ for a $k$-cell LFSR over $\mathbb{F}_{2}$ ); LFSRs are fast and easy to implement both in hardware and in software. However, sequences produced by LFSRs have linear dependencies that make easy to analyse the sequences to construct attacks on the whole cipher. To make output sequences of LFSRs more secure these linear dependencies must be destroyed by a properly chosen filter; this is the filter that carries the major cryptographical load making the whole cipher secure.

T-functions were found to be useful tools to design fast cryptographic primitives and ciphers based on usage of both arithmetic (addition, multiplication) and logical operations, see [46, 15, 16, 22, 21, 23, 26, 25, 24, 29, 37, 13, 40, 41, 45]. Loosely speaking, a T-function is a map of $k$-bit words into $k$-bit words such that each $i$-th bit of image depends only on low-order bits $0, \ldots, i$ of the pre-image. Various methods are known to construct bijective T-functions as well as transitive T-functions (the latter are the ones that produce sequences of the longest possible period, $2^{k}$ ), see $4,5,7,12,6,3,2,29,31,22,21,23,25,15$. Transitive T-functions have been considered as a candidate to replace LFSRs in keystream generators of stream ciphers, see e.g. 13, 46, 16, 24, 37, 40, since sequences produced by T-function-based keystream generators are proved to have a number of good cryptographic properties, e.g., high linear and 2-adic complexity, uniform distribution of subwords, etc., see [4, 28, 2, 50. Bijective T-functions can be used to design filter functions of stream ciphers, see [4, 2, 13].

2000 Mathematics Subject Classification. 94A60, 11S82, $11 \mathrm{~T} 71$.

Key words and phrases. T-function, bijectivity, transitivity, non-Archimedean ergodic theory, van der Put series, ergodicity, measure-preservation.

The authors were supported in part by grant of Linnaeus University Mathematical Modelling. The first of the authors was also supported by Russian Foundation for Basic Research grant No 12-01-00680-a and by Chinese Academy of Sciences visiting professorship for senior international scientists grant No 2009G2-11. 
The main purpose of this paper is to provide new criteria for bijectivity/transitivity of T-functions; so Theorems 6 and 7 are main results of the paper. In our opinion, these new criteria might be better applicable to T-functions that are represented via compositions of standard computer instructions on representation of T-functions via additions of some non-negative constants. What is important, the representation can be used to evaluate a T-function via a knapsack-like algorithm: Assuming the constants are stored in memory, to calculate a value of an arbitrary T-function on a $k$-bit word, one needs only not more than $k$ calls to memory and not more than $k-1$ additions modulo $2^{k}$ of $k$-bit numbers. Thus, representations of that sort could be used in design of various high-performance cryptographic primitives: keystream generators, filter functions, cipher combiners (Latin squares) and fast stream ciphers as a whole.

The representation is based on van der Put series, special convergent series from $p$-adic analysis; that is why the $p$-adic analysis (and $p$-adic ergodic theory) are main mathematical tools we use in the paper: To determine bijectivity/transitivity of a given T-function, we represent it via van der Put series (6) and apply accordingly Theorems 6 and/or 7 We stress that given a T-function combined from basic computer instructions, 'normally' it is easier to represent it via van der Put series than via Mahler series (or via coordinate functions $\psi_{i}$ to use criteria based on algebraic normal forms, see Subsection 2.4); moreover, once the T-function is represented via van der Put series, it is much faster to evaluate it compared to representation via Mahler series or via coordinate functions.

The van der Put series may also play an important role in a study of linear dependencies among coordinate sequences (the ones produced by the mentioned coordinate functions $\psi_{i}$ ) of a transitive T-function. Although given a 'randomly chosen' T-function, its coordinate sequences produced by $\psi_{i}$ and $\psi_{j}$ should be considered as 'independent' once $i \neq j$ (meaning the first half-periods of these sequences are independent Boolean vectors, cf. [4, Theorem 11.26]), this is not the case for large classes of transitive T-functions: There are linear relations between any two adjacent coordinate sequences in Klimov-Shamir T-functions [38, 39, in polynomials with integer coefficients [49, and in T-functions that are uniformly differentiable modulo 4, 43, 44]. The latter class is currently the largest known class where transitive T-functions exhibit linear relations between adjacent coordinate sequences. Therefore an important problem is to characterize transitive T-functions that exhibit no linear dependencies between adjacent coordinate sequences as the said linear relations may result in attacks against T-function-based ciphers, 44. Van der Put series might be an adequate tool in a study of the problem as with the use of the series one can handle 'non-smooth' T-functions (whence, the ones which are not uniformly differentiable modulo 4 ). We note however that the mentioned study is a future work whose subject is outside the scope of the current paper.

It is worth noticing here that the $p$-adic ergodic theory which is exploited in the current paper constitutes an important part of non-Archimedean (and wider, of algebraic) dynamics, a rapidly developing mathematical discipline that recently demonstrated its effectiveness in application to various sciences: computer science, cryptology, physics, molecular biology, cognitive sciences, genetics, artificial intelligence, image processing, numerical analysis and modelling, etc. Due to a huge number of papers in the area, we can mention only some monographs here to enable the interested reader to find relevant references therein: [4, 19, 18, 20]. 
The paper is organized as follows:

- In Section 2 we give a brief survey of non-Archimedean theory of Tfunctions: history, state of the art, main notions and results.

- In Section 3 we prove the said criteria for bijectivity/transitivity of Tfunctions in terms of van der Put series.

- Using the transitivity criterion, in Section 4 we give two examples of ergodic T-functions which are composed of additions and maskings. Also we explain how to use the bijectivity criterion in order to construct huge classes of large Latin squares and introduce a fast knapsack-like algorithm of evaluation of a T-function represented by van der Put series.

- We conclude in Section 5

\section{Non-ARCHIMEDEAN THEORY OF T-FUnCtIONS: BRIEF SURVEY}

In this Section, we introduce basics of what can be called the non-Archimedean approach to T-functions. For the full theory see monograph 4 or expository paper 2]. We start with a definition of a T-function and show that T-functions can be treated as continuous functions defined on and valued in the space of 2-adic integers. Therefore we introduce basics of 2-adic arithmetic and of 2-adic Calculus that we will need to state and prove our main results. There are many comprehensive monographs on $p$-adic numbers and $p$-adic analysis that contain all necessary definitions and proofs, see e.g. [27, 36, 42, or introductory chapters in [4; so further in the Section we introduce 2-adic numbers in a somewhat informal manner.

2.1. T-functions. An $n$-variate triangular function (a T-function for short) is a mapping

$$
\left(\alpha_{0}^{\downarrow}, \alpha_{1}^{\downarrow}, \alpha_{2}^{\downarrow}, \ldots\right) \mapsto\left(\Phi_{0}^{\downarrow}\left(\alpha_{0}^{\downarrow}\right), \Phi_{1}^{\downarrow}\left(\alpha_{0}^{\downarrow}, \alpha_{1}^{\downarrow}\right), \Phi_{2}^{\downarrow}\left(\alpha_{0}^{\downarrow}, \alpha_{1}^{\downarrow}, \alpha_{2}^{\downarrow}\right), \ldots\right),
$$

where $\alpha_{i}^{\downarrow} \in \mathbb{F}_{2}^{n}$ is a Boolean columnar $n$-dimensional vector; $\mathbb{F}_{2}=\{0,1\}$ is a 2element field, and

$$
\Phi_{i}^{\downarrow}:\left(\mathbb{F}_{2}^{n}\right)^{i+1} \rightarrow \mathbb{F}_{2}^{m}
$$

maps $(i+1)$ Boolean columnar $n$-dimensional vectors $\alpha_{0}^{\downarrow}, \ldots, \alpha_{i}^{\downarrow}$ to $m$-dimensional columnar Boolean vector $\Phi_{i}^{\downarrow}\left(\alpha_{0}^{\downarrow}, \ldots, \alpha_{i}^{\downarrow}\right)$. Accordingly, a univariate T-function $f$ is a mapping

$$
\left(\chi_{0}, \chi_{1}, \chi_{2}, \ldots\right) \stackrel{f}{\mapsto}\left(\psi_{0}\left(\chi_{0}\right) ; \psi_{1}\left(\chi_{0}, \chi_{1}\right) ; \psi_{2}\left(\chi_{0}, \chi_{1}, \chi_{2}\right) ; \ldots\right),
$$

where $\chi_{j} \in\{0,1\}$, and each $\psi_{j}\left(\chi_{0}, \ldots, \chi_{j}\right)$ is a Boolean function in Boolean variables $\chi_{0}, \ldots, \chi_{j}$. T-functions may be viewed as mappings from non-negative integers to non-negative integers: e.g., a univariate T-function $f$ sends a number with the base-2 expansion

$$
\chi_{0}+\chi_{1} \cdot 2+\chi_{2} \cdot 2^{2}+\cdots
$$

to the number with the base- 2 expansion

$$
\psi_{0}\left(\chi_{0}\right)+\psi_{1}\left(\chi_{0}, \chi_{1}\right) \cdot 2+\psi_{2}\left(\chi_{0}, \chi_{1}, \chi_{2}\right) \cdot 2^{2}+\cdots
$$

Further in the paper we refer to these Boolean functions $\psi_{0}, \psi_{1}, \psi_{2}, \ldots$ as coordinate functions of a T-function $f$. If we restrict T-functions to the set of all numbers whose base- 2 expansions are not longer than $k$, we sometimes refer these restrictions as T-functions on $k$-bit words. Thus, we may consider the restriction of a (univariate) T-function $f$ on $k$-bit words as a transformation $f \bmod 2^{k}$ of the 
residue ring $\mathbb{Z} / 2^{k} \mathbb{Z}$ modulo $2^{k}$ as every residue $r \in \mathbb{Z} / 2^{k} \mathbb{Z}$ can be associated to a base-2 expansion of a non-negative integer from $\left\{0,1, \ldots, 2^{k}-1\right\}$.

Important examples of T-functions are basic machine instructions:

- integer arithmetic operations (addition, multiplication,... );

- bitwise logical operations (OR, XOR, AND, NOT);

- some of their compositions (masking with mask $m, \operatorname{MASK}(\cdot, m)=\cdot \operatorname{AND} m$; $\ell$-bit shifts towards higher order bits, $\cdot 2^{\ell}$; reduction modulo $2^{k}, \cdot \bmod 2^{k}=$ - AND $\left.\left(2^{k}-1\right)\right)$.

Since obviously a composition of T-functions is a T-function (for instance, any polynomial with integer coefficients is a T-function), the T-functions are natural transformations of binary words that can be performed by digital computers. That is the main reason why T-functions have attracted attention of cryptographic community: T-functions may be used to construct new cryptographic primitives that are suitable for software implementations. For this purpose, given a T-function $f$ and a bitlength $k$, it is important to determine whether the mapping $f \bmod 2^{k}$ is bijective (that is, invertible) or transitive (that is, a permutation with a single cycle).

Although in cryptology the term "T-function" was suggested only in 2002, by Klimov and Shamir, see [22], in mathematics these mappings were known (however, under other names) long before 2002, and various effective criteria were proved to determine bijectivity and/or transitivity of T-functions. Some of these criteria were published in 1994 by the first author of the paper, see [5]; the criteria are based on 2-adic ergodic theory and use representation of a T-function $f$ in the form of Mahler series (5). However, in some cases to represent given T-function in this form may be a difficult mathematical task by itself; that is why a variety of criteria is needed to handle T-functions represented as compositions of various computer instructions (we give a brief survey of known criteria in Subsection 2.4).

2.2. Brief history of T-functions. As said, in cryptology the term "T-function" was suggested only in 2002, in mathematics the mappings we refer to as T-functions have being studied more than half a century.

In automata theory, Yablonsky et. al. studied the so-called determinate functions since 1950-th, see [47. Actually, a determinate function is a function that can be represented by an automaton: Consider an automaton with a binary input and binary output; then the automaton transforms each infinite input string of 0 -s and 1-s into infinite output string of 0-s and 1-s (we suppose that the initial state of the automaton is fixed). Note that every outputted $i$-th bit depends only on the inputted $i$-th bit and on the current state of the automaton. However, the current state depends only on the previous state and on the $(i-1)$-th input bit. Hence, for every $i=1,2, \ldots$, the $i$-th outputted bit depends only on bits $1,2, \ldots, i$ of the input string, and so the transformation of all infinite binary strings performed by the automaton is a T-function. The $p$-adic approach in automata theory goes back to the work of Lunts 35] of 1965.

We note here that Yablonsky and his succeeders were mostly interested in such properties of determinate functions as completeness of various systems of functions, various methods how to construct an automaton that represents the given function, etc. It is worth noticing also that determinate functions were studied in a more general setting, for arbitrary $K$-letter inputs/outputs, cf. [4] or recent works [10, 
11] and references therein. It is worth noticing here that in [10] (as well as in the current paper) van der Put series is the main tool.

T-functions were also studied in algebra, and also in much more general setting: T-functions are a special case of the so-called compatible mappings; namely, any T-function on $k$-bit words is a compatible mapping of a residue ring modulo $2^{k}$.

Recall that a transformation $f: A \rightarrow A$ of an algebraic system $A$ (that is, of a set endowed with operations $\omega \in \Omega$ ) is called compatible whenever $f$ agrees with all congruences of $A$; that is, $f(a) \sim f(b)$ whenever $a \sim b$, where $\sim$ is a congruence of $A$. Recall that a congruence $\sim$ is an equivalence relation that agrees with all operations $\omega$ of $A$ : $\omega\left(a_{1}, \ldots, a_{r}\right) \sim \omega\left(b_{1}, \ldots, b_{r}\right)$ whenever $a_{i} \sim b_{i}, i=1,2, \ldots, r$, $r$ is the arity of $\omega$. It is obvious that a compatible transformation of the residue ring $\mathbb{Z} / 2^{k} \mathbb{Z}$ modulo $2^{k}$ is a $\mathrm{T}$-function on $k$-bit words (under a natural one-to-one correspondence between $\mathbb{F}_{2}^{k}$ and $\mathbb{Z} / 2^{k} \mathbb{Z}$, when an $k$-bit word from $\mathbb{F}_{2}^{k}$ is considered as a base- 2 expansion of the corresponding integer): In the case when $A=\mathbb{Z} / 2^{k} \mathbb{Z}$ is a residue ring modulo $2^{k}$ the compatibility of $f$ yields

$$
a \equiv b \quad\left(\bmod 2^{s}\right) \Longrightarrow f(a) \equiv f(b) \quad\left(\bmod 2^{s}\right),
$$

for all $s \leq k$, which is merely an equivalent definition of a (univariate) T-function on $k$-bit words. For further results on compatible functions on rings and other algebras see monograph [32] and references therein.

Since the early 1990-th the non-Archimedean theory of T-functions emerged, which treated T-functions as continuous transformations of the space $\mathbb{Z}_{2}$ of 2-adic integers and studied corresponding dynamics. The first publications in this area were [5] and [12]; the importance of T-functions for pseudorandom generation and cryptology was explicitly stated in these papers as well. Within that theory, it was demonstrated that T-functions are continuous transformations with respect to 2 -adic metric, and that bijectivity (resp., transitivity) of T-functions correspond to measure preservation (resp., ergodicity) of these continuous transformations. This approach supplies a researcher with a number of effective tools from the nonArchimedean (actually, 2-adic) analysis to determine whether a given T-function is bijective or transitive, to study distribution and structure of output sequences, to construct wide classes of T-functions with prescribed properties, etc. We use this approach in the present paper.

We note that the theory was developed in a more general setting, for arbitrary prime $p$, and not necessarily for $p=2$, which corresponds to the case of T-functions. In the paper, we are mostly interested in the case $p=2$; however, we prove corresponding results for arbitrary prime $p$ where possible.

Last, but not least: T-functions under the name of triangular Boolean mappings were studied in the theory of Boolean functions. Within this theory there were obtained important criteria for invertibility/transitivity of a T-function, in terms of coordinate Boolean functions $\psi_{0}, \psi_{1}, \psi_{2}, \ldots$. The criteria belongs to mathematical folklore circulating at least since 1970-th among mathematicians dealing with the theory of Boolean functions; unfortunately the author of these important criteria is not known. To the best of our knowledge, the first quotation of these folklore criteria in literature occurred in 1994, see [5, Lemma 4.8]: As it is clearly marked in the mentioned paper, the said Lemma is just a re-statement of these well-known criteria. We reproduce these folklore criteria below, see Theorem 2 , 
2.3. T-functions, 2-adic integers, and $p$-adic analysis. As it follows directly from the definition, any T-function is well-defined on the set $\mathbb{Z}_{2}$ of all infinite binary sequences $\ldots \delta_{2}(x) \delta_{1}(x) \delta_{0}(x)=x$, where $\delta_{j}(x) \in \mathbb{F}_{2}=\{0,1\}, j=0,1,2, \ldots$. Arithmetic operations (addition and multiplication) with these sequences can be defined via standard "school-textbook" algorithms of addition and multiplication of natural numbers represented by base-2 expansions. Each term of a sequence that corresponds to the sum (respectively, to the product) of two given sequences can be calculated by these algorithms within a finite number of steps.

Thus, $\mathbb{Z}_{2}$ is a commutative ring with respect to the addition and multiplication defined in this manner. The ring $\mathbb{Z}_{2}$ is called the ring of 2-adic integers. The ring $\mathbb{Z}_{2}$ contains a subring $\mathbb{Z}$ of all rational integers: For instance, ...111 $=-1$.

Moreover, the ring $\mathbb{Z}_{2}$ contains all rational numbers that can be represented by irreducible fractions with odd denominators. For instance, $\ldots 01010101 \times \ldots 00011=$ $\ldots 111$, i.e., $\ldots 01010101=-1 / 3$ since $\ldots 00011=3$ and $\ldots 111=-1$.

Sequences with only a finite number of 1 -s correspond to non-negative rational integers in their base-2 expansions, sequences with only a finite number of 0 -s correspond to negative rational integers, while eventually periodic sequences (that is, sequences that become periodic from a certain place) correspond to rational numbers represented by irreducible fractions with odd denominators: For instance, $3=\ldots 00011,-3=\ldots 11101,1 / 3=\ldots 10101011,-1 / 3=\ldots 1010101$. So the $j$-th term $\delta_{j}(u)$ of the corresponding sequence $u \in \mathbb{Z}_{2}$ is merely the $j$-th digit of the base- 2 expansion of $u$ whenever $u$ is a non-negative rational integer, $u \in \mathbb{N}_{0}=$ $\{0,1,2, \ldots\}$.

What is important, the ring $\mathbb{Z}_{2}$ is a metric space with respect to the metrics (distance) $d_{2}(u, v)$ defined by the following rule: $d_{2}(u, v)=|u-v|_{2}=1 / 2^{n}$, where $n$ is the smallest non-negative rational integer such that $\delta_{n}(u) \neq \delta_{n}(v)$, and $d_{2}(u, v)=$ 0 if no such $n$ exists (i.e., if $u=v)$. For instance $d_{2}(3,1 / 3)=1 / 8$. The function $d_{2}(u, 0)=|u|_{2}$ is a 2-adic absolute value of the 2-adic integer $u$, and $\operatorname{ord}_{2} u=$ $-\log _{2}|u|_{2}$ is a 2 -adic valuation of $u$. Note that for $u \in \mathbb{Z}$ the valuation $\operatorname{ord}_{2} u$ is merely the exponent of the highest power of 2 that divides $u$ (thus, loosely speaking, $\operatorname{ord}_{2} 0=\infty$; so $|0|_{2}=0$ ). This means, in particular, that

$$
|a-b|_{2} \leq 2^{-k} \quad \text { if and only if } a \equiv b \quad\left(\bmod 2^{k}\right)
$$

for $a, b \in \mathbb{Z}$. Using this equivalence, one can expand the map mod $2^{k}$ (the reduction modulo $\left.2^{k}\right)$ to the whole space $\mathbb{Z}_{2}$, obtaining a T-function $\cdot \bmod 2^{k}=\cdot \operatorname{AND}\left(2^{k}-1\right)$ that is defined everywhere on $\mathbb{Z}_{2}$; so further we use both (equivalent) notations $|a-b|_{2} \leq 2^{-k}$ and $a \equiv b\left(\bmod 2^{k}\right)$ for arbitrary $a, b \in \mathbb{Z}_{2}$.

It is easy to see that the metric $d_{2}$ satisfies the strong triangle inequality:

$$
|a+b|_{2} \leq \max \left\{|a|_{2},|b|_{2}\right\},
$$

for all $a, b \in \mathbb{Z}_{2}$. Metric spaces of this kind are called ultrametric spaces, or nonArchimedean metrics spaces; the latter due to the fact that in ultrametric spaces the Archimedean Axiom does not hold.

Once the metric is defined, one defines notions of convergent sequences, limits, continuous functions on the metric space, and derivatives if the space is a commutative ring. For instance, with respect to the so defined metric $d_{2}$ on $\mathbb{Z}_{2}$ the sequence $1,3,7, \ldots, 2^{i}-1, \ldots$ (or, in base- 2 expansion, the sequence $1,11,111, \ldots$ ) tends to $-1=\ldots 111$. Bitwise logical operators (such as XOR, AND, ...) define continuous functions in two variables. 
Reduction modulo $2^{n}$ of a 2 -adic integer $v$, i.e., setting all terms of the corresponding sequence with indices greater than $n-1$ to zero (that is, taking the first $n$ digits in the representation of $v$ ) is just an approximation of a 2-adic integer $v$ by a rational integer with precision $1 / 2^{n}$ : This approximation is an $n$-digit positive rational integer $v$ AND $\left(2^{n}-1\right)$; the latter will be denoted also as $v \bmod 2^{n}$.

Actually a processor operates with approximations of 2-adic integers with respect to 2-adic metrics: When the overflow happens, i.e., when a number that must be written into an $n$-bit register consists of more than $n$ significant bits, the processor just writes only $n$ low order bits of the number into the register thus reducing the number modulo $2^{n}$. Thus, the accuracy of the approximation is defined by the bitlength of machine words of the processor.

What is the most important within the scope of the paper is that all T-functions are continuous functions of 2-adic variables, since all T-functions satisfy Lipschitz condition with a constant 1 with respect to the 2 -adic metrics, and vice versa.

Indeed, it is obvious that the function $f: \mathbb{Z}_{2} \rightarrow \mathbb{Z}_{2}$ satisfies the condition $\mid f(u)-$ $\left.f(v)\right|_{2} \leq|u-v|_{2}$ for all $u, v \in \mathbb{Z}_{2}$ if and only if $f$ is compatible, since the inequality $|a-b|_{2} \leq 1 / 2^{k}$ is just equivalent to the congruence $a \equiv b\left(\bmod 2^{k}\right)$. A similar property holds for multivariate T-functions. So we conclude:

$$
\text { T-functions }=\text { compatible functions }=1 \text {-Lipschitz functions }
$$

The observation we just have made implies that the non-Archimedean (namely, the 2-adic) analysis can be used in the study of T-functions. For instance, one can prove that the following functions satisfy Lipschitz condition with a constant 1 and thus are T-functions (so we can use them in compositions to construct PRNGs):

- subtraction, - : $(u, v) \mapsto u-v$;

- exponentiation, $\uparrow:(u, v) \mapsto u \uparrow v=(1+2 u)^{v}$, and in particular raising to negative powers, $u \uparrow(-v)=(1+2 u)^{-v}$;

- division, $/ /: u / / v=u \cdot(v \uparrow(-1))=u /(1+2 v)$.

We summarize:

- T-functions on $n$-bit words are approximations of 2-adic compatible functions (i.e., 1-Lipschitz functions) with precision $2^{-n}$ w.r.t. the 2 -adic metric: That is, a T-function on $n$-bit words is just a reduction modulo $2^{n}$ of a 2-adic 1-Lipschitz function.

- To study properties of T-functions one can use 2-adic analysis, since compatible functions are continuous w.r.t. the 2-adic metric.

- In addition to the basic machine instructions, to construct T-functions one can use also subtraction, division by an odd integer, raising an odd integer to a certain power.

All these considerations after proper modifications remain true for arbitrary prime $p$, and not only for $p=2$, thus resulting the notion of the $p$-adic integer and in respective $p$-adic analysis. For formal introduction to $p$-adic analysis, exact notions and results see any relevant book, e.g. [27, 36].

2.4. The 2-adic ergodic theory and bijectivity/transitivity of T-functions. Now we describe the connections between bijectivity/transitivity of T-functions and the 2-adic ergodic theory. We first recall some basic notions of dynamics and of ergodic theory (which is a part of dynamics). 
A dynamical system on a measure space $\mathbb{S}$ is a triple $(\mathbb{S} ; \mu ; f)$, where $\mathbb{S}$ is a set endowed with a measure $\mu$, and $f: \mathbb{S} \rightarrow \mathbb{S}$ is a measurable function; that is, an $f$ preimage of any measurable subset is a measurable subset. These basic definitions from dynamical system theory, as well as the following ones, can be found in [30; see also [17 as a comprehensive monograph on various aspects of dynamical systems theory.

A trajectory (or, orbit) is a sequence

$$
x_{0}, x_{1}=f\left(x_{0}\right), \ldots, x_{i}=f\left(x_{i-1}\right)=f^{i}\left(x_{0}\right), \ldots
$$

of points of the space $\mathbb{S}, x_{0}$ is called an initial point of the trajectory. If $F: \mathbb{S} \rightarrow \mathbb{T}$ is a measurable mapping to some other measurable space $\mathbb{T}$ with a measure $\nu$ (that is, if an $F$-preimage of any $\nu$-measurable subset of $\mathbb{T}$ is a $\mu$-measurable subset of $\mathbb{S})$, the sequence $F\left(x_{0}\right), F\left(x_{1}\right), F\left(x_{2}\right), \ldots$ is called an observable. Note that the trajectory formally looks like the sequence of states of a pseudorandom generator while the observable resembles the output sequence.

A mapping $F: \mathbb{S} \rightarrow \mathbb{Y}$ of a measure space $\mathbb{S}$ into a measure space $\mathbb{Y}$ endowed with probability measures $\mu$ and $\nu$, respectively, is said to be measure preserving (or, sometimes, equiprobable) whenever $\mu\left(F^{-1}(S)\right)=\nu(S)$ for each measurable subset $S \subset \mathbb{Y}$. In the case $\mathbb{S}=\mathbb{Y}$ and $\mu=\nu$, a measure preserving mapping $F$ is said to be ergodic whenever for each measurable subset $S$ such that $F^{-1}(S)=S$ one has either $\mu(S)=1$ or $\mu(S)=0$.

Recall that to define a measure $\mu$ on some set $\mathbb{S}$ we should assign non-negative real numbers to some subsets that are called elementary. All other measurable subsets are compositions of these elementary subsets with respect to countable unions, intersections, and complements.

Elementary measurable subsets in $\mathbb{Z}_{2}$ are balls $\mathbf{B}_{2^{-k}}(a)=a+2^{k} \mathbb{Z}_{2}$ of radii $2^{-k}$ centered at $a \in \mathbb{Z}_{2}$ (that is, co-sets with respect to the ideal $2^{k} \mathbb{Z}_{2}$ of the ring $\mathbb{Z}_{2}$, generated by $2^{k}$ ). To each ball we assign a number $\mu_{2}\left(\mathbf{B}_{2^{-k}}(a)\right)=1 / 2^{k}$. This way we define the probability measure $\mu_{2}$ on the space $\mathbb{Z}_{2}, \mu_{2}\left(\mathbb{Z}_{2}\right)=1$. The measure $\mu_{2}$ is a (normalized) Haar measure on $\mathbb{Z}_{2}$. The normalized Haar measure on $\mathbb{Z}_{2}^{n}$ can be defined in a similar manner.

To put it in other words, a ball $a+2^{k} \mathbb{Z}_{2}$ (of radius $2^{-k}$ ) is just a set of all 2-adic integers that are congruent to $a$ modulo $2^{k}$; that is, the set of all infinite binary words that have common initial prefix of length $k$ which coincides with the one of the (infinite) binary word $a$. The measure of this set is $\mu_{2}\left(a+2^{k} \mathbb{Z}_{2}\right)=2^{-k}$. For example, $\cdots * * * * * 0101=5+16 \cdot \mathbb{Z}_{2}=-1 / 3+16 \cdot \mathbb{Z}_{2}$ is a ball of radius (and of measure) $1 / 16$ centered at the point 5 (or, which is the same, at the point $-1 / 3$ ); all 2 -adic numbers that are congruent to 5 modulo 16 comprise this ball.

Note that the sequence $\left(s_{i}\right)_{i=0}^{\infty}$ of 2-adic integers is uniformly distributed (with respect to the measure $\mu_{2}$ on $\mathbb{Z}_{2}$ ) if and only if it is uniformly distributed modulo $2^{k}$ for all $k=1,2, \ldots$; That is, for every $a \in \mathbb{Z} / 2^{k} \mathbb{Z}$ relative numbers of occurrences of $a$ within initial segment of length $\ell$ of the sequence $\left(s_{i} \bmod 2^{k}\right)_{i=0}^{\infty}$ of residues modulo $2^{k}$ are asymptotically equal, i.e., $\lim _{\ell \rightarrow \infty} A(a, \ell) / \ell=1 / 2^{k}$, where $A(a, \ell)=\#\left\{s_{i} \equiv a\left(\bmod 2^{k}\right): i<\ell\right\}$, see [30] for details. Thus, strictly uniformly distributed sequences are uniformly distributed in the usual meaning of the theory of distributions of sequences. Of course, considerations of the above sort take place for arbitrary prime $p$, and not only in the case when $p=2$.

The following Theorem (which was announced in [7] and proved in [1]) holds: 
Theorem 1. For $m=n=1$, a 1-Lipschitz mapping $F: \mathbb{Z}_{p}^{n} \rightarrow \mathbb{Z}_{p}^{m}$ preserves the normalized Haar measure $\mu_{p}$ on $\mathbb{Z}_{p}$ (resp., is ergodic with respect to $\mu_{p}$ ) if and only if it is bijective (resp., transitive) modulo $p^{k}$ for all $k=1,2,3, \ldots$.

For $n \geq m$, the mapping $F$ preserves the measure $\mu_{p}$ if and only if it induces a balanced mapping of $\left(\mathbb{Z} / p^{k} \mathbb{Z}\right)^{n}$ onto $\left(\mathbb{Z} / p^{k} \mathbb{Z}\right)^{m}$, for all $k=1,2,3, \ldots$..

In other words, Theorem 1 yields that

- for a univariate T-function $f$, measure preservation is equivalent to bijectivity of $f \bmod 2^{k}$ for all $k \in \mathbb{N}$;

- for a multivariate $T$-function $F: \mathbb{Z}_{2}^{n} \rightarrow \mathbb{Z}_{2}^{m}, m \leq n$, measure preservation is equivalent to a balance of $F \bmod 2^{k}$ for all $k \in \mathbb{N}$;

- ergodicity of $F: \mathbb{Z}_{2}^{n} \rightarrow \mathbb{Z}_{2}^{n}$ is equivalent to transitivity of $F \bmod 2^{k}$ for all $k \in \mathbb{N}$.

This theorem implies in particular that whenever one chooses an ergodic T-function $f: \mathbb{Z}_{2} \rightarrow \mathbb{Z}_{2}$ as a state transition function of an automaton and a measure-preserving T-function $F:\left(\mathbb{Z} / 2^{k} \mathbb{Z}\right)^{n} \rightarrow\left(\mathbb{Z} / 2^{k} \mathbb{Z}\right)^{m}$ as an output function, both the sequence of states and output sequence of the automaton are uniformly distributed with respect to the Haar measure. This implies that reduction of these sequences modulo $2^{n}$ results in strictly uniformly distributed sequences of binary words. Note also that any number that is longer than a word bitlength of a computer, is reduced modulo $2^{n}$ automatically.

Thus, Theorem 1 points out a way to construct generators of uniformly distributed sequences from standard computer instructions. To construct such a generator, one must answer the following questions: What compositions of basic machine instructions are measure-preserving? are ergodic? Given a composition of basic machine instructions, is it measure-preserving? is it ergodic? These questions can be answered with the use of $p$-adic ergodic theory, see papers [5, 7, 12, 6, 3, 2, 1, 8, for complete theory and its applications to numerous sciences see monograph [4].

Now we recall two results from the 2 -adic ergodic theory. The first one is a 40year old folklore criteria of measure-preservation/ergodicity in terms of coordinate functions of a T-function.

2.4.1. Criteria based on algebraic normal forms. Recall that the algebraic normal form (the ANF for short) of the Boolean function $\psi_{j}\left(\chi_{0}, \ldots, \chi_{j}\right)$ is the representation of this function via $\oplus$ (addition modulo 2, that is, "exclusive or") and . (multiplication modulo 2, that is, "and", or conjunction). In other words, the ANF of the Boolean function $\psi$ is its representation in the form

$$
\psi\left(\chi_{0}, \ldots, \chi_{j}\right)=\beta \oplus \beta_{0} \chi_{0} \oplus \beta_{1} \chi_{1} \oplus \ldots \oplus \beta_{0,1} \chi_{0} \chi_{1} \oplus \ldots,
$$

where $\beta, \beta_{0}, \ldots \in\{0,1\}$. Recall also that the weight of the Boolean function $\psi_{j}$ in $(j+1)$ variables is the number of $(j+1)$-bit words that satisfy $\psi_{j}$; that is, the weight is the cardinality of the truth set of the Boolean function $\psi_{j}$.

Theorem 2 (Folklore). Let a univariate T-function $f$ be represented in the form (11). The T-function $f$ is measure-preserving iff for each $j=0,1, \ldots$ the Boolean function $\psi_{j}$ in Boolean variables $\chi_{0}, \ldots, \chi_{j}$ is linear with respect to the variable $\chi_{j}$; that is, $f$ is measure-preserving iff the ANF of each $\psi_{j}$ is of the form

$$
\psi_{j}\left(\chi_{0}, \ldots, \chi_{j}\right)=\chi_{j} \oplus \phi_{j}\left(\chi_{0}, \ldots, \chi_{j-1}\right),
$$

where $\phi_{j}$ is a Boolean function that does not depend on the variable $\chi_{j}$. 
The univariate T-function $f$ is ergodic iff, additionally, all Boolean functions $\phi_{j}$ are of odd weight. The latter takes place iff $\phi_{0}=1$, and the full degree of the Boolean function $\phi_{j}$ for $j \geq 1$ is exactly $j$, that is, the ANF of $\phi_{j}$ contains a monomial $\chi_{0} \cdots \chi_{j-1}$. Thus, $f$ is ergodic iff $\psi_{0}\left(\chi_{0}\right)=\chi_{0} \oplus 1$, and for $j \geq 1$ the ANF of each $\psi_{j}$ is of the form

$$
\psi_{j}\left(\chi_{0}, \ldots, \chi_{j}\right)=\chi_{j} \oplus \chi_{0} \cdots \chi_{j-1} \oplus \theta_{j}\left(\chi_{0}, \ldots, \chi_{j-1}\right)
$$

where the weight of $\theta_{j}$ is even; i.e., $\operatorname{deg} \theta_{j} \leq j-1$.

For the proof of the folklore Theorem see [4, Theorem 4.39], or [5] Lemma 4.8], or [50, Theorem 1].

Remark 1. Actually the bit-slice technique of Klimov and Shamir introduced in 22$]$ is just a re-statement of Theorem 2 .

We note that areas of applications of Theorem 2 are restricted: Given a Tfunction in a form of composition of basic computer instructions, most often it is infeasible to find its coordinate functions $\psi_{i}$. Thus, to determine with the use of that theorem whether a given composition of arithmetic and bitwise logical operators is bijective or transitive is possible only for relatively simple compositions like the mapping $x \mapsto x+x^{2}$ OR $C$ considered in [22]. The latter mapping is transitive modulo $2^{n}$ if and only if $C \equiv 5(\bmod 8)$ or $C \equiv 7(\bmod 8)$; see [4, Example 9.32], [8, Example 3.14], or [3. Example 4.9] for the proof based on Theorem 2.

Earlier in 1999 Kotomina 29] applied Theorem[2] to prove the following statement resulting in the so called add-xor generators, which are extremely fast: The Tfunction

$$
f(x)=\left(\ldots\left(\left(\left(\left(x+c_{0}\right) \mathrm{XOR} d_{0}\right)+c_{1}\right) \mathrm{XOR} d_{1}\right)+\cdots\right.
$$

is transitive modulo $2^{n}(n \geq 2)$ if and only if it is transitive modulo 4.

The following proposition, whose proof is also based on Theorem 2, gives a method to construct new invertible T-functions (respectively, T-functions with a single cycle property), out of given T-functions:

Proposition 1 (see [8, 3] and [4, Proposition 9.29]). Let $F$ be an $(n+1)$-variate T-function such that for all $z_{1}, \ldots, z_{n}$ the T-function $F\left(x, z_{1}, \ldots, z_{n}\right)$ is measurepreserving with respect to the variable $x$. Then the composition

$$
F\left(f(x), 2 g_{1}(x), \ldots, 2 g_{n}(x)\right)
$$

is measure-preserving for arbitrary T-functions $g_{1}, \ldots, g_{n}$ and any invertible $T$ function $f$.

Moreover, if $f$ is ergodic, then $f(x+4 g(x)), f(x \mathrm{XOR}(4 g(x))), f(x)+4 g(x)$, and $f(x)$ XOR $(4 g(x))$ are ergodic, for arbitrary T-function $g$.

Although Theorem 2 can be applied to determine invertibility/single cycle property of some T-functions, it is highly doubtful that one can prove, with the use of Theorem 2 only, that, e.g., the following function $f$ is a T-function that is ergodic (it is!):

$$
\begin{gathered}
f(x)=2+\frac{x}{3}+\frac{1}{3^{x}}+2\left(\frac{\left(x^{2}+2 x\right) \operatorname{XOR}(1 / 3)}{2 x+3}\right)^{\frac{(x+1) \operatorname{And}(1 / 5)}{1-2 x}}+ \\
2 \operatorname{NOT}\left(\left(\frac{\left(x^{2}-1\right) \operatorname{XOR}(1 / 3)}{2 x+1}\right)^{\frac{x \operatorname{AND}(1 / 5)}{5-2 x}}\right) .
\end{gathered}
$$


Therefore we need more delicate tools than Theorem 2 to study complicated compositions of basic machine instructions. These tools are provided by $p$-adic analysis and $p$-adic ergodic theory. The first one of the said tools are Mahler series.

2.4.2. Criteria based on Mahler series. It is not difficult to see that every mapping $f: \mathbb{N}_{0} \rightarrow \mathbb{Z}_{p}$ (or, respectively, $f: \mathbb{N}_{0} \rightarrow \mathbb{Z}$ ) admits one and only one representation in the form of so-called Mahler interpolation series

$$
f(x)=\sum_{i=0}^{\infty} a_{i}\left(\begin{array}{l}
x \\
i
\end{array}\right)
$$

where $\left(\begin{array}{c}x \\ i\end{array}\right)=x(x-1) \cdots(x-i+1) / i$ ! for $i=1,2, \ldots$, and $\left(\begin{array}{l}x \\ 0\end{array}\right)=1 ; a_{i} \in \mathbb{Z}_{p}$ (respectively, $\left.a_{i} \in \mathbb{Z}\right), i=0,1,2, \ldots$. This statement can be easily proved directly, substituting successively $x=0,1,2, \ldots$ to $(5)$ and solving the corresponding equation with unknown $a_{x}$.

Foremost, if $f$ is uniformly continuous on $\mathbb{N}_{0}$ with respect to the $p$-adic distance, $f$ can be uniquely expanded to a uniformly continuous function on $\mathbb{Z}_{p}$ since $\mathbb{Z}$ is dense in $\mathbb{Z}_{p}$. Hence the interpolation series for $f$ converges uniformly on $\mathbb{Z}_{p}$. The following is true (see e.g. [36]): The series $f(x)=\sum_{i=0}^{\infty} a_{i}\left(\begin{array}{c}x \\ i\end{array}\right),\left(a_{i} \in \mathbb{Z}_{p}\right.$, $i=0,1,2, \ldots)$ converges uniformly on $\mathbb{Z}_{p}$ if and only if $\lim _{i \rightarrow \infty}^{p} a_{i}=0$, where $\lim ^{p}$ is a limit with respect to the $p$-adic distance; hence uniformly convergent series defines a uniformly continuous function on $\mathbb{Z}_{p}$.

The following theorem holds:

Theorem 3 (see [5, 7] and [4, Theorem 3.53]). The function $f: \mathbb{Z}_{p} \rightarrow \mathbb{Z}_{p}$ represented by (5) is compatible if and only if

$$
a_{i} \equiv 0 \quad\left(\bmod p^{\left\lfloor\log _{p} i\right\rfloor}\right)
$$

for all $i=2,3,4, \ldots$. (Here and after for a real $\alpha$ we denote $\lfloor\alpha\rfloor$ the integral part of $\alpha$, i.e., the nearest to $\alpha$ rational integer that is not larger than $\alpha$.)

Remark 2. We remind that in the case $p=2$ compatible functions are $T$-functions, and vice versa.

Remark 3. Note that the number $\left\lfloor\log _{p} i\right\rfloor$ for $i=1,2,3, \ldots$ has a very natural meaning: it is the number of digits in a base-p expansion of $i$, decreased by 1 . That is, $\left\lfloor\log _{p} i\right\rfloor$ is a bitlength of $i$, decreased by 1 . So within the context of the paper it is reasonable to assume that $\left\lfloor\log _{p} 0\right\rfloor=0$.

Now we can give general characterization of measure-preserving (resp., ergodic) T-functions:

Theorem 4 (see [5, 12] and [4, Theorem 4.40]). A map $f: \mathbb{Z}_{2} \rightarrow \mathbb{Z}_{2}$ is a measure preserving $T$-function if and only if it can be represented as

$$
f(x)=c_{0}+x+\sum_{i=1}^{\infty} c_{i} 2^{\left\lfloor\log _{2} i\right\rfloor+1}\left(\begin{array}{l}
x \\
i
\end{array}\right) .
$$

The map $f$ is an ergodic T-function if and only if it can be represented as

$$
f(x)=1+x+\sum_{i=1}^{\infty} c_{i} 2^{\left\lfloor\log _{2}(i+1)\right\rfloor+1}\left(\begin{array}{l}
x \\
i
\end{array}\right),
$$

where $c_{0}, c_{1}, c_{2} \ldots \in \mathbb{Z}_{2}$. 
Using the identity $\Delta\left(\begin{array}{c}x \\ i\end{array}\right)=\left(\begin{array}{c}x \\ i-1\end{array}\right)$, where $\Delta$ is a difference operator, $\Delta u(x)=$ $u(x+1)-u(x)$, we immediately deduce from Theorems 3 and 4 the following easy method to construct a measure preserving or ergodic T-function out of an arbitrary T-function:

Corollary 1 ([7, also [4, Theorem 4.44]). Every ergodic (respectively, every measure preserving) T-function $f: \mathbb{Z}_{2} \rightarrow \mathbb{Z}_{2}$ can be represented as

$$
f(x)=1+x+2 \cdot \Delta g(x)
$$

(respectively, as $f(x)=d+x+2 \cdot g(x)$ ) for a suitable $d \in \mathbb{Z}_{2}$ and a suitable $T$-function $g: \mathbb{Z}_{2} \rightarrow \mathbb{Z}_{2}$; and vice versa, every function $f$ of the above form is, accordingly, ergodic or measure-preserving T-function.

Remark 4. Ergodicity of the T-function (4) can be proved with the use of Corollary 1

2.4.3. Why new techniques are needed. Theorems 2 and 4 give methods to construct measure-preserving/ergodic T-functions from arithmetic and bitwise logical computer instructions; these methods may be too difficult (or even impossible) to use when a T-function is a composition of both arithmetic and bitwise logical operations like masking (MASK $(x, c)=x \operatorname{AND} c$; i.e. when the composition includes $i$-th bit value functions $\delta_{i}(x)$. For instance, not speaking of more complicated compositions, it is quite difficult to determine measure preservation/ergodicity even in the simplest case when $f$ is a linear combination of $\delta_{i}(x)$ : for the corresponding (rather involved) proof see [4, Theorem 9.20] or [3]. However, functions of this sort are easy to implement in software and hardware; moreover, they were already used in some ciphers, see e.g. 13. There are other techniques in $p$-adic ergodic theory than the already mentioned ones, like the methods that exploit uniform differentiability (we do not mention the ones in the paper; see [4, Section 4.6] about these). However, T-functions that include compositions of bitwise logical operations are rarely uniformly differentiable.

That is one of reasons why we need new techniques to handle T-functions of this sort. These new techniques are based on representation of T-functions in the form of van der Put series.

The other reason is that using the van der Put representations turns out to be a general way to speed-up T-function-based cryptographic algorithms via timememory trade-offs since actually evaluation of a T-function represented by van der Put series uses just memory calls and integer summations.

It should be stressed however that compared to the known criteria the new ones are not superior in all cases, for all T-functions: An answer to the question which of the criteria is better to use in order to determine bijectivity/transitivity of a given T-function strongly depends on a composition of the T-function. In some cases a particular criterion just works better than others. For instance, to determine when a linear combination of $\delta_{i}(x)$ is transitive one may use either criteria based on Mahler series (Theorem 4), or criteria based on ANFs of coordinate functions (Theorem 2) or new criteria based on van der Put series (Theorem 7 ). Then, exploiting the criterion based on Mahler series results in a long involved proof (cf. 4, Theorem 9.20]) while application of the ANF-based criterion seemingly results in a shorter one, whereas the use of the new criterion implies a very short proof, cf. Example 1. We believe (and give some evidence in Section 4) that the new criteria are most suitable for T-functions whose compositions involve numerous bitwise 
logical instructions. However, the ANF-based criteria may be as effective (or even better) if a T-function is a relatively short composition of bitwise logical and/or arithmetic instructions, whereas the criteria based on Mahler series are seemingly more useful for 'classical-shaped' functions (2-adic exponential functions, rational functions, etc.); and for 'smooth' T-functions it is reasonable to try first criteria that exploit differentiability, cf. [4, Section 4.6; Subsection 9.2.2]. Moreover, results of the current paper, after being announced (without proofs) in 9], already have stimulated development of ergodic theory in the ring $\mathbb{F}_{2}[[X]]$ of formal power series over a two-element field $\mathbb{F}_{2}$, see recent paper [34. Maybe the criteria developed in the latter paper can also be applied to determine bijectivity/transitivity of certain T-functions as well since the metric space of all infinite binary sequences with the metric $d_{2}$ can also be treated as the metric space $\mathbb{F}_{2}[[X]]$ (rather than the metric space $\mathbb{Z}_{2}$ ). We believe that no universal 'superior' criterion exists; nonetheless, every new criterion enriches researchers' toolbox making it more diverse and therefore giving more flexibility when determining bijectivity/transitivity of a given T-function.

2.5. Van der Put series. Now we recall the definition and some properties of van der Put series, see e.g. 36, 42 for details. Given a continuous function $f: \mathbb{Z}_{p} \rightarrow \mathbb{Z}_{p}$, there exists a unique sequence $B_{0}, B_{1}, B_{2}, \ldots$ of $p$-adic integers such that

$$
f(x)=\sum_{m=0}^{\infty} B_{m} \chi(m, x)
$$

for all $x \in \mathbb{Z}_{p}$, where

$$
\chi(m, x)= \begin{cases}1, & \text { if }|x-m|_{p} \leq p^{-n} \\ 0, & \text { otherwise }\end{cases}
$$

and $n=1$ if $m=0 ; n$ is uniquely defined by the inequality $p^{n-1} \leq m \leq p^{n}-1$ otherwise. The right side series in (6) is called the van der Put series of the function $f$. Note that the sequence $B_{0}, B_{1}, \ldots, B_{m}, \ldots$ of van der Put coefficients of the function $f$ tends $p$-adically to 0 as $m \rightarrow \infty$, and the series converges uniformly on $\mathbb{Z}_{p}$. Vice versa, if a sequence $B_{0}, B_{1}, \ldots, B_{m}, \ldots$ of $p$-adic integers tends $p$-adically to 0 as $m \rightarrow \infty$, then the series in the right part of (6) converges uniformly on $\mathbb{Z}_{p}$ and thus defines a continuous function $f: \mathbb{Z}_{p} \rightarrow \mathbb{Z}_{p}$.

The number $n$ in the definition of $\chi(m, x)$ has a very natural meaning; it is just the number of digits in a base- $p$ expansion of $m \in \mathbb{N}_{0}$ : As said (see Remark [3),

$$
\left\lfloor\log _{p} m\right\rfloor=(\text { the number of digits in a base- } p \text { expansion for } m)-1 \text {; }
$$

therefore $n=\left\lfloor\log _{p} m\right\rfloor+1$ for all $m \in \mathbb{N}_{0}$ (recall that we assume $\left\lfloor\log _{p} 0\right\rfloor=0$ ).

Note that the coefficients $B_{m}$ are related to the values of the function $f$ in the following way: Let $m=m_{0}+\ldots+m_{n-2} p^{n-2}+m_{n-1} p^{n-1}$ be a base- $p$ expansion for $m$, i.e., $m_{j} \in\{0, \ldots, p-1\}, j=0,1, \ldots, n-1$ and $m_{n-1} \neq 0$, then

$$
B_{m}= \begin{cases}f(m)-f\left(m-m_{n-1} p^{n-1}\right), & \text { if } m \geq p ; \\ f(m), & \text { if otherwise. }\end{cases}
$$


It is worth noticing also that $\chi(m, x)$ is merely a characteristic function of the ball $\mathbf{B}_{p^{-\left\lfloor\log _{p} m\right\rfloor-1}}(m)=m+p^{\left\lfloor\log _{p} m\right\rfloor-1} \mathbb{Z}_{p}$ of radius $p^{-\left\lfloor\log _{p} m\right\rfloor-1}$ centered at $m \in \mathbb{N}_{0}$ :

$$
\chi(m, x)=\left\{\begin{array}{ll}
1, & \text { if } x \equiv m \quad\left(\bmod p^{\left\lfloor\log _{p} m\right\rfloor+1}\right) ; \\
0, & \text { if otherwise }
\end{array}= \begin{cases}1, & \text { if } x \in \mathbf{B}_{p^{-\left\lfloor\log _{p} m\right\rfloor-1}}(m) \\
0, & \text { if otherwise }\end{cases}\right.
$$

\section{MAin RESUlts}

In this Section we prove criteria for measure-preservation/ergodicity for a $\mathrm{T}$ function in terms of van der Put coefficients of the T-function. However, we start with a van der Put coefficients based criterion for compatibility of a continuous $p$-adic map $\mathbb{Z}_{p} \rightarrow \mathbb{Z}_{p}$. In the case $p=2$ the criterion yields necessary and sufficient conditions for a map $\mathbb{Z}_{2} \rightarrow \mathbb{Z}_{2}$ to be a T-function.

3.1. The compatibility criterion in terms of van der Put coefficients. We first prove the compatibility criterion for arbitrary map $\mathbb{Z}_{p} \rightarrow \mathbb{Z}_{p}$ represented by van der Put series.

Theorem 5 (Compatibility criterion). Let a function $f: \mathbb{Z}_{p} \rightarrow \mathbb{Z}_{p}$ be represented via van der Put series (6); then $f$ is compatible (that is, satisfies the $p$-adic Lipschitz condition with a constant 1) if and only if $\left|B_{m}\right|_{p} \leq p^{-\left\lfloor\log _{p} m\right\rfloor}$ for all $m=0,1,2, \ldots$.

In other words, $f$ is compatible if and only if it can be represented as

$$
f(x)=\sum_{m=0}^{\infty} p^{\left\lfloor\log _{p} m\right\rfloor} b_{m} \chi(m, x),
$$

for suitable $b_{m} \in \mathbb{Z}_{p} ; m=0,1,2, \ldots$. In particular, every T-function $f: \mathbb{Z}_{2} \rightarrow \mathbb{Z}_{2}$ can be represented as

$$
f(x)=\sum_{m=0}^{\infty} 2^{\left\lfloor\log _{2} m\right\rfloor} b_{m} \chi(m, x),
$$

where $b_{m} \in \mathbb{Z}_{2}, m=0,1,2, \ldots$; and vice versa, the series (10) defines a T-function.

Proof of Theorem 5. To prove the necessity of conditions, take $m \in \mathbb{N}_{0}$ and consider its base- $p$-expansion $m=m_{0}+\ldots+m_{n-1} p^{n-1}$. Here $m_{j} \in\{0, \ldots, p-1\}, m_{n-1} \neq 0$, and $n=\left\lfloor\log _{p} m\right\rfloor+1$. As

$$
m_{0}+\ldots+m_{n-2} p^{n-2} \equiv m_{0}+\ldots+m_{n-2} p^{n-2}+m_{n-1} p^{n-1} \quad\left(\bmod p^{n-1}\right) ;
$$

then

$$
f\left(m_{0}+\ldots+m_{n-2} p^{n-2}\right) \equiv f\left(m_{0}+\ldots+m_{n-1} p^{n-1}\right) \quad\left(\bmod p^{n-1}\right)
$$

by the compatibility of $f$. From the latter congruence in view of (7) it follows that $B_{m} \equiv 0\left(\bmod p^{n-1}\right)$ for $m \geq p$; so $\left|B_{m}\right| \leq p^{-\left\lfloor\log _{p} m\right\rfloor}$.

Now we prove the sufficiency of conditions. As $\left|B_{m}\right|_{p} \leq p^{-\left\lfloor\log _{p} m\right\rfloor}$, the sequence $B_{0}, B_{1}, \ldots$ tends $p$-adically to 0 as $m \rightarrow \infty$ and so the function $f$ is continuous. Hence while proving that $|f(x)-f(y)|_{p} \leq|x-y|_{p}$ for $x, y \in \mathbb{Z}_{p}$ we may assume that $x, y \in \mathbb{N}_{0}$ as $\mathbb{N}_{0}$ is dense in $\mathbb{Z}_{p}$. Moreover, by same reasons to prove that $f$ satisfies $p$-adic Lipschitz condition with a constant 1 it suffices only to prove that given $x \in \mathbb{N}_{0}$ and $h, n \in \mathbb{N},\left|f\left(x+h p^{n}\right)-f(x)\right|_{p} \leq p^{-n}$. 
Let $h=h_{0}+h_{1} p+\ldots+h_{\ell} p^{\ell}$ be a base- $p$ expansion of $h \in \mathbb{N}$, and let $n_{0}<$ $n_{1}<n_{2}<\ldots<n_{k}$ be all indices in the latter base- $p$ expansion such that $h_{n_{0}}, h_{n_{1}}, \ldots, h_{n_{k}}$ are nonzero; so $h=h_{n_{0}} p^{n_{0}}+h_{n_{1}} p^{n_{1}}+\ldots+h_{n_{k}} p^{n_{k}}$. Now in view of (7) we have that

$$
f\left(x+h p^{n}\right)=f(x)+\left[f\left(x+p^{n} h_{n_{0}} p^{n_{0}}\right)-f(x)\right]+
$$

$$
\begin{array}{r}
\sum_{j=1}^{k}\left[f\left(x+p^{n}\left(h_{n_{0}} p^{n_{0}}+\cdots+h_{n_{j}} p^{n_{j}}\right)\right)-f\left(x+p^{n}\left(h_{n_{0}} p^{n_{0}}+\cdots+h_{n_{j-1}} p^{n_{j-1}}\right)\right)\right]= \\
f(x)+\sum_{j=0}^{k} B_{x+h_{n_{0}} p^{n+n_{0}+\cdots+h_{n_{j}}} p^{n+n_{j}}}
\end{array}
$$

However, by our assumption,

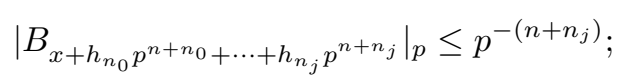

so (11) implies that $\left|f\left(x+h p^{n}\right)-f(x)\right|_{p} \leq p^{-n}$ due to the strong triangle inequality that holds for the $p$-adic absolute value, cf. (3).

3.2. The measure-preservation criteria for T-functions, in terms of the van der Put coefficients. Now we prove two criteria of measure-preservation for T-functions.

Theorem 6. Let $f: \mathbb{Z}_{2} \rightarrow \mathbb{Z}_{2}$ be a T-function represented via van der Put series (6). The T-function $f$ is measure-preserving if and only if the following conditions hold simultaneously:

(i) $B_{0}+B_{1} \equiv 1(\bmod 2)$;

(ii) $\left|B_{m}\right|_{2}=2^{-\left\lfloor\log _{2} m\right\rfloor}, m=2,3, \ldots$.

Proof. By Corollary 1 the T-function $f$ is measure-preserving if and only if it can be represented in the form $f(x)=d+x+2 g(x)$, where $g: \mathbb{Z}_{2} \rightarrow \mathbb{Z}_{2}$ is a T-function and $d \in \mathbb{Z}_{2}$. Now, given $g(x)=\sum_{m=0}^{\infty} \tilde{B}_{m} \chi(m, x)$, the van der Put series for the Tfunction $g$, we find van the der Put coefficients of the function $f(x)=d+x+2 g(x)$.

As the van der Put series of the T-function $t(x)=x$ is

$$
t(x)=\sum_{m=1}^{\infty} 2^{\left\lfloor\log _{2} m\right\rfloor} \chi(m, x),
$$

and as $d=d \cdot \chi(0, x)+d \cdot \chi(1, x)$, we get:

$$
\begin{aligned}
& f(x)=\left(d+2 \tilde{B}_{0}\right) \chi(0, x)+\left(1+d+2 \tilde{B}_{1}\right) \chi(1, x)+ \\
& \sum_{m=2}^{\infty}\left(2^{\left\lfloor\log _{2} m\right\rfloor}+2 \tilde{B}_{m}\right) \chi(m, x) .
\end{aligned}
$$

This proves necessity of conditions of the Theorem since $\left|\tilde{B}_{m}\right|_{2} \leq 2^{-\left\lfloor\log _{2} m\right\rfloor}$ by Theorem $\left[5\right.$ and hence $\left|2^{\left\lfloor\log _{2} m\right\rfloor}+2 \tilde{B}_{m}\right|_{2}=2^{-\left\lfloor\log _{2} m\right\rfloor}$ by the strong triangle inequality (3).

To prove sufficiency of the conditions, we note that the condition $\left|B_{m}\right|_{2}=$ $2^{-\left\lfloor\log _{2} m\right\rfloor}$ implies that $B_{m}=2^{\left\lfloor\log _{2} m\right\rfloor}+2 \tilde{B}_{m}$ for suitable $\tilde{B}_{m} \in \mathbb{Z}_{2}$, where

$$
\left|\tilde{B}_{m}\right|_{2} \leq 2^{-\left\lfloor\log _{2} m\right\rfloor}
$$


and the condition $B_{0}+B_{1} \equiv 1(\bmod 2)$ implies that $B_{0}=d+2 \tilde{B}_{0}$ and $B_{1}=$ $1+d+2 \tilde{B}_{1}$ for suitable $d, \tilde{B}_{0}, \tilde{B}_{1} \in \mathbb{Z}_{2}$. Now from equations (13) and (12) it follows that $f(x)=d+x+2 g(x)$, where $g(x)=\sum_{m=0}^{\infty} \tilde{B}_{m} \chi(m, x)$ is a T-function by Theorem 5 in view of inequality (14). Thus, $f$ is measure-preserving by Corollary 1 .

From Theorems 5 and 6 we deduce now the following

Corollary 2. A map $f: \mathbb{Z}_{2} \rightarrow \mathbb{Z}_{2}$ is a measure-preserving T-function if and only if it can be represented as

$$
f(x)=b_{0} \chi(0, x)+b_{1} \chi(1, x)+\sum_{m=2}^{\infty} 2^{\left\lfloor\log _{2} m\right\rfloor} b_{m} \chi(m, x),
$$

where $b_{m} \in \mathbb{Z}_{2}$, and the following conditions hold simultaneously

(i) $b_{0}+b_{1} \equiv 1(\bmod 2)$;

(ii) $b_{m} \equiv 1(\bmod 2), m=2,3,4 \ldots$.

3.3. The ergodicity criteria for T-functions, in terms of the van der Put coefficients. In this subsection, we prove the following criterion of ergodicity for T-functions:

Theorem 7. A T-function $f: \mathbb{Z}_{2} \rightarrow \mathbb{Z}_{2}$ is ergodic if and only if it can be represented as

$$
f(x)=b_{0} \chi(0, x)+b_{1} \chi(1, x)+\sum_{m=2}^{\infty} 2^{\left\lfloor\log _{2} m\right\rfloor} b_{m} \chi(m, x)
$$

for suitable $b_{m} \in \mathbb{Z}_{2}$ that satisfy the following conditions:

(i) $b_{0} \equiv 1(\bmod 2)$;

(ii) $b_{0}+b_{1} \equiv 3(\bmod 4)$;

(iii) $\left|b_{m}\right|_{2}=1, m \geq 2$;

(iv) $b_{2}+b_{3} \equiv 2(\bmod 4)$;

(v) $\sum_{m=2^{n-1}}^{2^{n}-1} b_{m} \equiv 0(\bmod 4), n \geq 3$.

To prove the Theorem, we need the following Lemma:

Lemma 1 (48]). Let $f: \mathbb{Z}_{2} \rightarrow \mathbb{Z}_{2}$ be a T-function represented by van der Put series (6). Then $f$ is ergodic if and only there exists a sequence $a_{0}, a_{1}, \ldots$ of 2 -adic integers such that

$$
B_{m}= \begin{cases}1+2\left(a_{1}-a_{0}\right), & \text { if } m=0 ; \\ 2\left(1+a_{0}+2 a_{2}-a_{1}\right), & \text { if } m=1 ; \\ 2^{n-1}+2^{n} a_{m+1}-2^{n} a_{m}, & \text { if } 2^{n-1} \leq m<2^{n}-1, n \geq 2 \\ 2^{n-1}+2^{n+1} a_{2^{n}}-2^{n} a_{2^{n}-1}-2^{n} a_{2^{n-1}}, & \text { if } m=2^{n}-1, n \geq 2 .\end{cases}
$$

Proof. By Corollary 1, a T-function $f$ is ergodic if and only if it can be represented as $f(x)=1+x+2(g(x+1)-g(x))$, where $g(x)$ is a suitable T-function. That is, by Theorem 5 ,

$$
g(x)=a_{0} \chi(0, x)+\sum_{m=1}^{\infty} 2^{n_{m}-1} a_{m} \chi(m, x)=\sum_{m=0}^{\infty} \tilde{B}_{m} \chi(m, x),
$$


for suitable $a_{0}, a_{1}, a_{2} \ldots \in \mathbb{Z}_{2}, \tilde{B}_{0}, \tilde{B}_{1}, \tilde{B}_{2}, \ldots \in \mathbb{Z}_{2}$ (here $n_{m}=\left\lfloor\log _{2} m\right\rfloor+1, m=$ $1,2,3, \ldots)$.

Now, to prove necessity of conditions of the Lemma, we just need to express the van der Put coefficients of the function $f$ via the coefficients of the function $g$. First, we do this for the van der Put coefficients $\bar{B}_{m}$ of the T-function

$$
g(x+1)=\sum_{m=0}^{\infty} \bar{B}_{m} \chi(m, x) .
$$

If $m>1$ then by (7) $\bar{B}_{m}=g(m+1)-g(m+1-q(m))$ where $q(m)=\delta_{n_{m}-1}(m) 2^{n_{m}-1}$. If $m \neq 2^{n_{m}}-1$ then $q(m)=q(m+1)$, therefore $\bar{B}_{m}=g(m+1)-g(m+1-q(m+1))=$ $\tilde{B}_{m+1}=2^{n_{m}-1} a_{m+1}$ by (14) as $n_{m}=n_{m+1}$ in this case. If $m=2^{n_{m}}-1$ then $\bar{B}_{m}=g\left(2^{n_{m}}\right)-g\left(2^{n_{m}-1}\right)$ as $q\left(2^{n_{m}}-1\right)=2^{n_{m}-1}$. As $\tilde{B}_{2^{n}}=g\left(2^{n}\right)-g(0)$ and $\tilde{B}_{2^{n-1}}=g\left(2^{n-1}\right)-g(0)$ by (14), we conclude that $\bar{B}_{2^{n}-1}=\tilde{B}_{2^{n}}-\tilde{B}_{2^{n-1}}$. Finally, the coefficients $\bar{B}_{0}, \bar{B}_{1}$ can be found directly from (17): $\bar{B}_{0}=g(1)=\tilde{B}_{1}$, $\bar{B}_{1}=g(2)=\tilde{B}_{0}+\tilde{B}_{2}$. Now we can find the van der Put coefficients $\hat{B}_{m}$ of the function $2(g(x+1)-g(x))$; they are:

$$
\hat{B}_{m}= \begin{cases}2\left(\tilde{B}_{1}-\tilde{B}_{0}\right), & \text { if } m=0 ; \\ 2\left(\tilde{B}_{0}+\tilde{B}_{2}-\tilde{B}_{1}\right), & \text { if } m=1 ; \\ 2\left(\tilde{B}_{m+1}-\tilde{B}_{m}\right), & \text { if } 2^{n-1} \leq m<2^{n}-1, n \geq 2 ; \\ 2\left(\tilde{B}_{m+1}-\tilde{B}_{m}-\tilde{B}_{\frac{m+1}{2}}\right), & \text { if } m=2^{n}-1, n \geq 2 .\end{cases}
$$

As $\chi(0, x)+\chi(1, x)=1$ for all $x \in \mathbb{Z}_{2}$, from (10) we derive the van der Put expansion for the function $x+1$; namely,

$$
x+1=\chi(0, x)+2 \chi(1, x)+\sum_{m=2}^{\infty} 2^{n_{m}-1} \chi(m, x) .
$$

From (14) we have that $\tilde{B}_{0}=a_{0}, \tilde{B}_{m}=2^{n-1} a_{m}$ when $2^{n-1} \leq m \leq 2^{n}-1$, $n=1,2, \ldots$. Now combining the latter expressions with (19) and (17) we conclude that the van der Put coefficients $B_{m}$ of the function $f(x)=1+x+2(g(x+1)-g(x))$ are of the form (15).

To prove sufficiency of conditions of the Lemma we just remark that the above argument shows that given expressions (15) for the van der Put coefficients of the function $f$ we can represent the T-function $f$ in the form $f(x)=1+x+2(g(x+$ 1) $-g(x))$ where the van der Put expansion for $g$ is given by (14). That is, the function $g$ is a T-function by Theorem 5 therefore the T-function $f$ is ergodic by Corollary 1 .

Now we are able to prove the following Proposition which actually is a criterion of ergodicity for T-functions, in terms of van der Put coefficients:

Proposition 2. Let $f: \mathbb{Z}_{2} \rightarrow \mathbb{Z}_{2}$ be a T-function represented by the van der Put series (6). Then $f$ is ergodic if and only if the following conditions are satisfied simultaneously:

(i) $B_{0} \equiv 1(\bmod 2)$;

(ii) $B_{0}+B_{1} \equiv 3(\bmod 4)$;

(iii) $\left|B_{m}\right|_{2}=2^{-(n-1)}, n \geq 2,2^{n-1} \leq m<2^{n}-1$;

(iv) $\left|\sum_{m=2^{n-1}}^{2^{n}-1}\left(B_{m}-2^{n-1}\right)\right|_{2} \leq 2^{-(n+1)}, n \geq 2$. 
Proof. By Lemma 1 if the T-function $f$ is ergodic then its van der Put coefficients $B_{m}$ can be expressed in the form (15), for suitable $a_{0}, a_{1}, a_{2}, \ldots \in \mathbb{Z}_{2}$. From (15) by direct calculation we easily prove that conditions (i)-(iv) of the Proposition are true.

By Lemma 1 to prove sufficiency of conditions of the Proposition we must find a sequence of 2-adic integers $a_{0}, a_{1}, a_{2}, \ldots$ such that relations (15) for the van der Put coefficients $B_{n}$ hold. Take arbitrarily $a_{0}, a_{1} \in \mathbb{Z}_{2}$ so that

$$
a_{1}-a_{0}=\frac{B_{0}-1}{2}
$$

(cf. the first equation from (15) and condition (i) of the Proposition); then put

$$
a_{2}=\frac{B_{1}+2\left(a_{1}-a_{0}\right)-2}{4}=\frac{B_{1}+B_{0}-3}{4}
$$

(cf. the second equation from (15D). Note that $a_{2} \in \mathbb{Z}_{2}$ due to the condition (ii) of the Proposition. We construct $a_{3}, a_{4}, a_{5}, \ldots \in \mathbb{Z}_{2}$ inductively. Denote

$$
\check{B}_{m}=\frac{B_{m}-2^{n-1}}{2^{n}}
$$

where $n=\left\lfloor\log _{2} m\right\rfloor+1, m \geq 3$; then $\check{B}_{m} \in \mathbb{Z}_{2}$ by condition (iii). Given $a_{2^{n-1}} \in \mathbb{Z}_{2}$, for $\alpha=1,2, \ldots, 2^{n-1}-1$ put

$$
\begin{aligned}
a_{2^{n-1}+\alpha} & =a_{2^{n-1}}+\sum_{m=2^{n-1}}^{2^{n-1}+\alpha-1} \check{B}_{m}, \\
a_{2^{n}} & =a_{2^{n-1}}+\frac{1}{2} \sum_{m=2^{n-1}}^{2^{n}-1} \check{B}_{m} .
\end{aligned}
$$

Then $a_{2^{n-1}+\alpha} \in \mathbb{Z}_{2}$ by condition (iii) of the Proposition; and $a_{2^{n}} \in \mathbb{Z}_{2}$ by condition (iv). Therefore all $a_{0}, a_{1}, a_{2}, \ldots$ are in $\mathbb{Z}_{2}$.

Now solving system of equations (20),(21), (22), (23), (24) with respect to unknowns $B_{m}, m=0,1,2,3, \ldots$, we see that the van der Put coefficients $B_{m}$ satisfy conditions (15) of Lemma 1 Therefore $f$ is ergodic.

Now we are able to prove Theorem 7

Proof of Theorem 7 . Consider the van der Put expansion (6) of the T-function $f$;

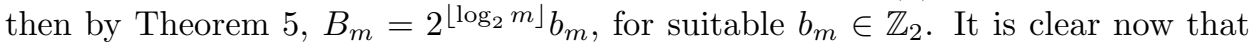
conditions (i), (ii) and (iii) of Proposition 2 are equivalent respectively to conditions (i), (ii) and (iii) of Theorem 7

Take $2^{n-1} \leq m<2^{n}, n \geq 2$; thus $B_{m}=2^{n-1} b_{m}$. Then condition (iv) of Proposition 2 is equivalent to the congruence $\sum_{m=2^{n-1}}^{2^{n}-1}\left(B_{m}-2^{n-1}\right) \equiv 0\left(\bmod 2^{n+1}\right)$; which is equivalent to the congruence $2^{n-1} \sum_{m=2^{n-1}}^{2^{n}-1}\left(b_{m}-1\right) \equiv 0\left(\bmod 2^{n+1}\right)$ as $B_{m}=2^{n-1} b_{m}$. However, the latter congruence is equivalent to the congruence $\sum_{m=2^{n-1}}^{2^{n}-1}\left(b_{m}-1\right) \equiv 0(\bmod 4)$ which in turn is equivalent either to the congruence $\sum_{m=2^{n-1}}^{2^{n}-1} b_{m} \equiv 0(\bmod 4)($ when $n \geq 3)$ or to the congruence $\sum_{m=2^{n-1}}^{2^{n}-1} b_{m} \equiv 2$ $(\bmod 4)$ (when $n=2)$. However, the latter two congruences are respectively conditions (v) and (iv) of Theorem 7. 


\section{Applications}

In this Section we consider some applications of the above criteria: We give a new (and short!) proof of ergodicity of a known ergodic T-function which is used in a filter of $\mathrm{ABC}$ stream cipher from [13, then we prove ergodicity of a more complicated T-function (the latter result is new). After that we explain how to use Theorem 6 (the bijectivity criterion) in order to construct huge classes of large Latin squares. Finally we present a knapsack-like algorithm for fast computation of arbitrary T-function that use only integer additions and calls to memory.

4.1. Examples of ergodic T-functions with masking. In this Subsection we consider two example of ergodic T-functions constructed from additions, multiplications and masking (i.e., the instruction $\operatorname{MASK}(x, c)=x$ AND $c$ ). Thus, being implemented as computer programs both these T-functions are fast enough.

The T-function from Example 1 is used to construct a filter in ABC stream cipher [13] however, the proof of its ergodicity (which is based on Mahler series and Theorem 4) is highly technical and complicated, see e.g. [4, Theorem 9.20] or 3. Although a shorter proof might be given with the use of ANF-based criteria (Theorem 21), below we give a very short proof by applying the ergodicity criteria in terms of van der Put series, cf. Theorem 7

Let $x=\chi_{0}+\chi_{1} \cdot 2+\ldots+\chi_{k} \cdot 2^{k}+\cdots$ be a 2-adic representation of $x \in \mathbb{Z}_{2}$; remind that we denote $\delta_{k}(x)=\chi_{k}$ (cf. beginning of Subsection 2.3). In other words, the value of the function $\delta_{k}: \mathbb{Z}_{2} \rightarrow \mathbb{F}_{2}$ at the point $x \in \mathbb{Z}_{2}$ is the $k$-th binary digit of the base-2 expansion of $x$; so $\sum_{k=0}^{\infty} 2^{k} \delta_{k}(x)=\sum_{k=0}^{\infty} \chi_{k} \cdot 2^{k}=x$. Note that $\delta_{k}(x)=1$ if and and only if $x$ is congruent modulo $2^{k+1}$ to either of the numbers $2^{k}, 2^{k}+1, \ldots, 2^{k+1}-1 ;$ so

$$
\delta_{k}(x)=\sum_{m=2^{k}}^{2^{k+1}-1} \chi(m, x),
$$

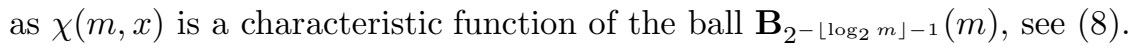

Example 1. Given a sequence $c, c_{0}, c_{1}, c_{2}, \ldots$ of 2-adic integers, the series

$$
c+\sum_{i=0}^{\infty} c_{i} \delta_{i}(x)
$$

defines an ergodic T-function $f: \mathbb{Z}_{2} \rightarrow \mathbb{Z}_{2}$ if and only if the following conditions hold simultaneously:

(i) $c \equiv 1(\bmod 2)$;

(ii) $c_{0} \equiv 1(\bmod 4)$;

(iii) $\left|c_{i}\right|_{2}=2^{-i}$, for $i=1,2,3, \ldots$.

Indeed, substituting (25) to (26) we obtain the series $c+\sum_{i=0}^{\infty} c_{i} \sum_{m=2^{i}}^{2^{i+1}-1} \chi(m, x)$; so the van der Put coefficients are: $B_{0}=c, B_{1}=c+c_{0}, B_{m}=c_{\left\lfloor\log _{2} m\right\rfloor}$ for $m \geq 2$. Now from condition (i) of Proposition 2 we have that $c \equiv 1(\bmod 2)$; however, from condition (ii) of Proposition 2 we have that $2 c+c_{0} \equiv 3(\bmod 4)$ which gives us that $c_{0} \equiv 1(\bmod 4)$. Condition (iii) of Proposition 2 is equivalent to the condition $\left|c_{i}\right|_{2}=2^{i}$ for $i \geq 1$. Due to these three conditions, condition (iv) of Proposition 2 is satisfied since $\sum_{m=2^{i}}^{2^{i+1}-1}\left(c_{i}-2^{i}\right)=2^{i} c_{i}-2^{2 i} \equiv 0\left(\bmod 2^{i+2}\right)$ for $i \geq 1$. This ends the proof. 
Note that under conditions of Example1, the T-function $f(x)=c+\sum_{i=0}^{\infty} c_{i} \delta_{i}(x)$ can be expressed via operations of integer addition, integer multiplication by constants, and operation of masking, $\operatorname{MASK}\left(x, 2^{i}\right)=x$ AND $2^{i}=2^{i} \delta_{i}(x)$ : As $c_{i}=2^{i} d_{i}$ for suitable $d_{i} \in \mathbb{Z}_{2}, i=0,1,2, \ldots$, by conditions (ii)-(iii), we have that $f(x)=$ $c+\sum_{i=0}^{\infty} d_{i} \cdot \operatorname{MASK}\left(x, 2^{i}\right)$; so the corresponding T-function $\bar{f}=f \bmod 2^{k}$ on $k$ bit words is $\bar{f}(x)=c+\sum_{i=0}^{k-1} d_{i} \cdot \operatorname{MASK}\left(x, 2^{i}\right)$.

Note that in the just considered example the coefficients $c, c_{0}, c_{1}, \ldots$ do not depend on $x$. Now we consider a more complicated T-function of this sort where the coefficients depend on $x$. As the least non-negative residue modulo $2^{k}$ is a special case of MASK instruction, $x \bmod 2^{k}=\operatorname{MASK}\left(x, 2^{k}-1\right)$, the T-function from Example 2 can be expressed via integer additions, multiplications, and masking.

Example 2. The following T-function $f$ is ergodic on $\mathbb{Z}_{2}$ :

$$
f(x)=1+\delta_{0}(x)+6 \delta_{1}(x)+\sum_{k=2}^{\infty}\left(1+2\left(x \bmod 2^{k}\right)\right) 2^{k} \delta_{k}(x) .
$$

To prove the assertion we calculate van der Put coefficients $B_{m}$. For $m \in\{0,1\}$ we have:

(i) $B_{0}=f(0)=1$

(ii) $B_{1}=f(1)=2$

Given $m=m_{0}+2 m_{1}+\ldots+2^{n-2} m_{n-2}+2^{n-1}$, denote $m=m+2^{n-1}, n_{m}=n=$ $\left\lfloor\log _{2} m\right\rfloor+1$. Then, we calculate the van der Put coefficients for the case $n_{m}=2$. As $m=m_{0}+2$ in this case, we see that

$$
B_{m_{0}+2}=f\left(m_{0}+2\right)-f\left(m_{0}\right)=6
$$

so $B_{2}=B_{3}=6$.

Now we proceed with calculations of $B_{m}$ for the case $n_{m}=n \geq 3$ :

$$
\begin{aligned}
B_{m} & =f\left(\dot{m}+2^{n-1}\right)-f(\dot{m})=1+\delta_{0}\left(\dot{m}+2^{n-1}\right)+6 \cdot \delta_{1}\left(\dot{m}+2^{n-1}\right)+ \\
& +\sum_{k=2}^{n-1} 2^{k}\left(1+2\left(\left(\dot{m}+2^{n-1}\right) \bmod 2^{k}\right)\right) \cdot \delta_{k}\left(\dot{m}+2^{n-1}\right)-f(\dot{m})= \\
& =f(\dot{m})+2^{n-1}(1+2 \dot{m})-f(\dot{m}) .
\end{aligned}
$$

So we conclude that if $n_{m} \geq 3$ then

$$
B_{m}=2^{n-1}(1+2 \dot{m})
$$

where $\dot{m}=m_{0}+2 m_{1}+\ldots+2^{n-2} m_{n-2}$. Finally we get:

(i) $B_{0}=1 \equiv 1(\bmod 2)$;

(ii) $B_{0}+B_{1}=1+2 \equiv 3(\bmod 4)$;

(iii) $\left|B_{m}\right|_{2}=\left|2^{n_{m}-1}(1+2 m)\right|_{2}=2^{-\left(n_{m}-1\right)}$ for $n_{m} \geq 3$, and $\left|B_{2}\right|_{2}=\left|B_{3}\right|_{2}=|6|_{2}=2^{-1}, n_{2}=n_{3}=2$. 
(iv) For $n_{m}=2$, we have that $\left|\left(B_{2}-2\right)+\left(B_{3}-2\right)\right|_{2}=2^{-(2+1)}$; and for $n_{m}=$ $n \geq 3$, we have that

$$
\begin{gathered}
\left|\sum_{m=2^{n-1}}^{2^{n}-1}\left(B_{m}-2^{n-1}\right)\right|_{2}=\left|\sum_{\dot{m}=0}^{2^{n-1}-1}\left(B_{\dot{m}+2^{n-1}}-2^{n-1}\right)\right|_{2}= \\
\left|\sum_{\dot{m}=0}^{2^{n-1}-1}\left(2^{n-1}(1+2 \dot{m})-2^{n-1}\right)\right|_{2}=\left|2^{n} \sum_{\dot{m}=0}^{2^{n-1}-1} \dot{m}\right|_{2}= \\
\left|2^{n} \cdot \frac{\left(1+2^{n-1}-1\right)}{2} \cdot\left(2^{n-1}-1\right)\right|_{2} \leq 2^{-(n+1)} .
\end{gathered}
$$

Therefore, under conditions of Example 2 the T-function $f$ is ergodic by Proposition 2

4.2. Latin squares. In this Subsection we explain how one may use the bijectivity criterion (Theorem 6) to construct Latin squares of order $2^{\ell}$. We recall that a Latin square of order $P$ is a $P \times P$ matrix containing $P$ distinct symbols (usually denoted by $0,1, \ldots, P-1$ ) such that each row and column of the matrix contains each symbol exactly once. Latins squares are used in numerous applications: For games (recall sudoku), for private communication networks (password distribution), in coding theory, in cryptography (e.g., as stream cipher combiners), etc., see, e.g., monographs [14, 33.

There is no problem to construct one Latin square (a circulant matrix serves an obvious example), a problem is how to write a software that produces a number of large Latin squares; however, this is only a part of the problem. Another part of the problem is that in some constraint environments (e.g., in smart cards) the whole matrix can not be stored in memory: Given two numbers $a, b \in\{0,1, \ldots, P-1\}$, the software must calculate the $(a, b)$-th entry of the matrix on-the-fly.

A number of methods have been developed in order to construct Latin squares, see e.g. the monographs we refer above; however, not all of the methods provide solution to the said problem since the methods are based on mappings which are somewhat slow if implemented in software (as, e.g., are polynomials over large finite fields). Therefore new methods that are based on 'fast' computer instructions are needed. Methods of the latter sort have been developed by using the 2-adic ergodic theory, see [4. Section 8.4] where the said theory is applied to construct Latin squares as well as pairs of orthogonal Latin squares. The methods of the mentioned monograph are based on differentiability of $p$-adic mappings; by using Theorem 6, methods based on van der Put series can also be developed. We illustrate the general idea by a simple example.

A Latin square of order $P$ is just a bivariate mapping $F:(\mathbb{Z} / P \mathbb{Z})^{2} \rightarrow \mathbb{Z} / P \mathbb{Z}$ which is bijective with respect to either variable. Therefore, given a pair of bijective (measure-preserving) T-functions

$$
f(x)=\sum_{m=0}^{\infty} 2^{\left\lfloor\log _{2} m\right\rfloor} b_{m} \chi(m, x) \text { and } \bar{f}(y)=\sum_{m=0}^{\infty} 2^{\left\lfloor\log _{2} m\right\rfloor} \bar{b}_{m} \chi(m, y)
$$


whose van der Put coefficients satisfy Corollary 2, the function

$$
F(x, y)=\left(\sum_{m=0}^{\infty} 2^{\left\lfloor\log _{2} m\right\rfloor}\left(b_{m} \chi(m, x)+\bar{b}_{m} \chi(m, y)\right)\right) \bmod p^{\ell}
$$

is a Latin square of order $2^{\ell}$. The idea can be developed further; however, this implies expanding of the apparatus of van der Put series to the multivariate case. Development of the corresponding theory can be a subject of a future work but now it is out of scope of the current paper. We note only that in order to obtain really fast performance of the corresponding software, methods of fast evaluation of T-functions are needed. We introduce a method of that kind in the next Subsection.

4.3. Fast computation of T-functions. In this Subsection we demonstrate how by using the van der Put representation of a T-function one could speed-up evaluation of the T-function via time-memory trade-offs.

Let a T-function $f$ be represented via van der Put series (6); then the respective T-function on $k$-bit words is

$$
\bar{f}=f \bmod 2^{k}=\sum_{m=0}^{2^{k}-1} B_{m} \chi(m, x)
$$

by Theorem 5 , see (10). Arrange coefficients $B_{m}, m=0,1, \ldots, 2^{k}-1$ into array $B(f)=\left[B_{m}: m=0,1, \ldots, 2^{k}-1\right]$; so the address of the coefficient $B_{m}$ is $m, m=$ $0,1, \ldots, 2^{k}-1$. From (27) and (8) it follows that the value $\bar{f}(x)$ of the T-function $f$ at $x \in\left\{0,1, \ldots, 2^{k}-1\right\}$ is equal to the sum modulo $2^{k}$ of coefficients $B_{m}$ for $m=x \bmod 2, x \bmod 4, \ldots, x \bmod 2^{k}$; so to calculate the output $\bar{f}(x)$ given input $x \in\left\{0,1, \ldots, 2^{k}-1\right\}$ one needs $k-1$ additions modulo $2^{k}$ and $k$ calls to memory. To calculate $\bar{f}(x)$ the following procedure may be used (note that $\operatorname{MASK}\left(x, 2^{j}-1\right)=$ $\left.x \bmod 2^{j}\right)$ :

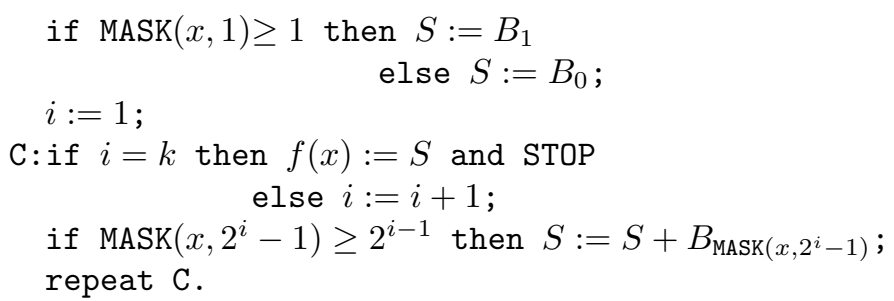

It can be easily seen that to compute $f(x)$ the procedure uses $k$ memory calls to retrieve relevant coefficients $B_{m}, k$ compare instructions $\geq / \geq$ of integers, $k$ maskings MASK, and $k-1$ integer additions. Note that if necessary the compare routine $\operatorname{MASK}\left(x, 2^{i}-1\right) \geq 2^{i-1}$ may be replaced with the routine to determine whether $\operatorname{MASK}\left(x, 2^{i-1}\right)$ is 0 or not; however this doubles the total number of maskings. Note also that given arbitrary measure-preserving (respectively, ergodic) T-function $f$, the array $B(f)$ consists of $2^{k}$ integers which may be too large in practical cases. If so, arrays where most entries $B_{m}$ are $2^{\left\lfloor\log _{2} m\right\rfloor}$ may be used then (cf. condition (ii) of Theorem 6 and condition (iii) of Proposition 2, respectively): In this case, most entries must not necessarily be kept in memory, they can be calculated on-the-fly instead, by a suitable fast routine. In connection with the issue it would be interesting to study other cryptographical properties of measure-preserving/ergodic T-functions whose van der Put coefficients comprise arrays of this kind. 


\section{Conclusion}

In the paper, we present new criteria for a T-function to be bijective (that is, measure-preserving) or transitive (that is, ergodic). In the proofs techniques from non-Archimedean ergodic theory are used: The new criteria are based on representation of a T-function via van der Put series, special series from $p$-adic analysis. We note that the criteria are not 'globally' superior to other known criteria (e.g., the ones based on Mahler series or on ANFs of coordinate functions): being necessary and sufficient conditions for the bijectivity/transitivity of T-functions, all the criteria (speaking rigorously) are equivalent one to another. However, some criteria are easier to apply to some particular types of T-functions than the other criteria. In the paper we give an evidence that the criteria based on van der Put series are most suitable to determine bijectivity/transitivity of a T-function whose composition includes machine instructions like, e.g., masking. We also use the van der Put series to construct a knapsack-like algorithm for fast evaluation of T-functions via time-memory trade-offs.

\section{REFERENCES}

[1] V. Anashin, Ergodic Transformations in the Space of $p$-adic Integers, in: p-adic Mathematical Physics. 2-nd Int'l Conference (Belgrade, Serbia and Montenegro 15-21 September 2005) (Andrei Yu. Khrennikov, Zoran Rakić and Igor V. Volovich, eds.), AIP Conference Proceedings 826, pp. 3-24, American Institute of Physics, Melville, New York, 2006.

[2] V. Anashin, Non-Archimedean Theory of T-Functions, in: Proc. Advanced Study Institute Boolean Functions in Cryptology and Information Security, NATO Sci. Peace Secur. Ser. D Inf. Commun. Secur. 18, pp. 33-57, IOS Press, Amsterdam, 2008.

[3] V. Anashin, Non-Archimedean Ergodic Theory and Pseudorandom Generators, The Computer Journal 53 (2010), 370-392.

[4] V. Anashin and A. Khrennikov, Applied Algebraic Dynamics, de Gruyter Expositions in Mathematics 49, Walter de Gruyter GmbH \& Co., Berlin-N.Y., 2009.

[5] V. S. Anashin, Uniformly distributed sequences of p-adic integers, Mathematical Notes 55 (1994), 109-133.

[6] V. S. Anashin, Uniformly distributed sequences in computer algebra, or how to constuct program generators of random numbers, J. Math. Sci. 89 (1998), 1355-1390.

[7] V. S. Anashin, Uniformly distributed sequences of $p$-adic integers, II, Discrete Math. Appl. 12 (2002), 527-590.

[8] V. S. Anashin, Pseudorandom number generation by p-adic ergodic transformations, Available from http://arxiv.org/abs/cs.CR/0401030 January 2004.

[9] V. S. Anashin, A. Yu. Khrennikov and E. I. Yurova, Characterization of ergodicity of $p$-adic dynamical systems by using van der Put basis, Doklady Mathematics 83(2011), 306-308.

[10] Vladimir Anashin, Automata finiteness criterion in terms of van der Put series of automata functions, p-Adic Numbers, Ultrametric Analysis and Applications 4 (2012), 151-160.

[11] Vladimir Anashin, The non-Archimedean theory of discrete systems, Math. Comp. Sci. (accepted for publication; preprint available from http://arxiv.org/abs/1112.5096)

[12] Vladimir Anashin, Uniformly distributed sequences over $p$-adic integers, in: Number theoretic and algebraic methods in computer science. Proceedings of the Int'l Conference (Moscow, June-July, 1993) (I. Shparlinsky A. J. van der Poorten and H. G. Zimmer, eds.), pp. 1-18, World Scientific, 1995.

[13] Vladimir Anashin, Andrey Bogdanov and Ilya Kizhvatov, ABC: A New Fast Flexible Stream Cipher, Version 3, eSTREAM, Report, 2005, Available from http://www.ecrypt.eu.org/stream/p2ciphers/abc/abc_p2.pdf

[14] J. Dénes and A. D. Keedwell, Latin squares, North-Holland, Amsterdam, 1991.

[15] J. Hong, D. Lee, Y. Yeom and D. Han, A new class of single cycle T-functions, in: Fast Software Encryption, Lect. Notes Comp. Sci. 3557, pp. 68-82, Springer-Verlag, 2005. 
[16] Jin Hong, Dong Hoon Lee, Yongjin Yeom and Daewan Han, T-function Based Stream Cipher TSC-3, eSTREAM, Report no. 2005/031, 2005, Available from http://www.ecrypt.eu.org/stream/ciphers/tsc3/tsc3.pdf

[17] A. Katok and B. Hasselblatt, Introduction to the Modern Theory of Dynamical Systems, Cambridge University Press, 1998.

[18] A. Yu. Khrennikov, Non-Archimedean Analysis: Quantum Paradoxes, Dynamical Systems and Biological Models, Kluwer Academic Publishers, Dordrecht, 1997.

[19] A. Yu. Khrennikov, Information dynamics in cognitive, psychological, social, and anomalous phenomena, Kluwer Academic Publishers, Dordreht, 2004.

[20] A. Yu. Khrennikov and M. Nilsson, p-adic deterministic and random dynamics, Kluver Academic Publ., Dordrecht etc., 2004.

[21] A. Klimov and A. Shamir, Cryptographic Applications of T-functions, in: Selected Areas in Cryptography, 3006, pp. 248-261, 2003.

[22] A. Klimov and A. Shamir, A new class of invertible mappings, in: Cryptographic Hardware and Embedded Systems 2002 (B.S.Kaliski Jr.et al., ed.), Lect. Notes in Comp. Sci 2523, pp. 470-483, Springer-Verlag, 2003.

[23] A. Klimov and A. Shamir, New cryptographic primitives based on multiword T-functions, in: Fast Software Encryption, 2004.

[24] A. Klimov and A. Shamir, The TF-i family of stream ciphers, Handout distributed at: The State of the Art of Stream Ciphers-SASC, 2004.

[25] Alexander Klimov, Applications of T-functions in Cryptography, Ph.D. thesis, Weizmann Institute of Science, 2005, Available from http://www.wisdom.weizmann.ac.il/ ask/

[26] Alexander Klimov and Adi Shamir, New Applications of T-Functions in Block Ciphers and Hash Functions, in: Fast Software Encryption (Henri Gilbert and Helena Handschuh, eds.), Lecture Notes in Computer Science 3557, pp. 18-31, Springer, 2005.

[27] N. Koblitz, p-adic numbers, $p$-adic analysis, and zeta-functions, second ed, Graduate texts in math. 58, Springer-Verlag, 1984.

[28] N. Kolokotronis, Cryptographic Properties of Nonlinear Pseudorandom Number Generators, Designs, Codes and Cryptography 46 (2008), 353-363.

[29] L. Kotomina, Fast nonlinear congruential generators, Master's thesis, Russian State University for the Humanities, Moscow, 1999, In Russian.

[30] L. Kuipers and H. Niederreiter, Uniform Distribution of Sequences, John Wiley \& Sons, N.Y. etc., 1974.

[31] M. V. Larin, Transitive polynomial transformations of residue class rings, Discrete Mathematics and Applications 12 (2002), 141-154.

[32] Hans Lausch and Wilfried Nöbauer, Algebra of Polynomials, North-Holl. Publ. Co, American Elsevier Publ. Co, 1973.

[33] C. F. Laywine and G. L. Mullen, Discrete mathematics using Latin squares, John Wiley \& Sons, Inc., New York, 1998.

[34] Dongdai Lin, Tao Shi and Zifeng Yang, Ergodic theory over $\mathbb{F}_{2}[[X]]$, Finite Fields Appl. 18(2012), 473-491.

[35] A. G. Lunts, The $p$-adic apparatus in the theory of finite automata, Problemy Kibernetiki 14 (1965), 17-30, In Russian.

[36] K. Mahler, p-adic numbers and their functions, Cambridge Univ. Press, 1981, (2nd edition).

[37] Alexander Maximov, A New Stream Cipher Mir-1, eSTREAM, Report no. 2005/017, 2005, Available from http://www.ecrypt.eu.org/stream

[38] Håvard Molland and Tor Helleseth, A linear weakness in the Klimov-Shamir T-function, in: Proc. 2005 IEEE Int. Symp. on Information Theory, pp. 1106-1110, 2005.

[39] Håvard Molland and Tor Helleseth, Linear properties in T-functions, IEEE Trans. Inf. Theory 52 (2006), 5151-5157.

[40] Dukjae Moon, Daesung Kwon, Daewan Han, Jooyoung Lee, Gwon Ho Ryu, Dong Wook Lee, Yongjin Yeom and Seongtaek Chee, T-function Based Stream Cipher TSC-4, eSTREAM, Report no. 2006/024, 2006, Available from http://www.ecrypt.eu.org/stream/papersdir/2006/024.pdf

[41] Sean O'Neil, Benjamin Gittins and Howard Landman, VEST, eSTREAM, Report, 2006, Available from http://www.ecrypt.eu.org/stream/vestp2.html

[42] W. H. Schikhof, Ultrametric calculus, Cambridge University Press, 1984. 
[43] Tao Shi, Vladimir Anashin and Dongdai Lin, Linear Weaknesses in T-functions, in: SETA 2012 (T. Helleseth and J. Jedwab., eds.), Lect. Notes Comp. Sci. 7280, pp. 279-290, SpringerVerlag, 2012.

[44] T. Shi, V. Anashin and D. Lin, Linear Relation on General Ergodic T-Function, http://arxiv.org/abs/1111.4635v1

[45] Synaptic Laboratories Limited, The VEST Cryptosystem for Semiconductors, Available from http://www.vestciphers.com/en/index.html

[46] Kai-Thorsten Wirt, ASC-A Stream Cipher with Built-In MAC Functionality, in: Proc. World Acad. Sci. Engineering and Technology, 23, 2007.

[47] S. V. Yablonsky, Basic notions of cybernetics, Problems of Cybernetics, Fizmatgiz, 1959, (in Russian).

[48] E. I. Yurova, Van der Put basis and p-adic dynamics, P-Adic Numbers, Ultrametric Analysis, and Applications, 2 (2010), 175-178.

[49] Jin-Song Wang and Wen-Feng Qi, Linear equation on polynomial single cycle T-function, in: Inscrypt 2007 (Dingyi Pei et al., ed.) Lect. Notes Comp. Sci. 4490, pp. 256-270, Springer Verlag, 2008.

[50] Wenying Zhang and Chuan-Kun Wu, The Algebraic Normal Form, Linear Complexity and k-Error Linear Complexity of Single-Cycle T-function, in: SETA 2006 (G. Gong et al., ed.), Lect. Notes Comp. Sci. 4086, pp. 391-401, Springer-Verlag, 2006. 